\title{
The light-fermion contribution to the exact Higgs-gluon form factor in QCD
}

\author{
Robert V. Harlander, ${ }^{a}$ Mario Prausa ${ }^{b}$ and Johann Usovitsch ${ }^{c}$ \\ ${ }^{a}$ Institute for Theoretical Particle Physics and Cosmology, \\ RWTH Aachen University, Aachen D-52056, Germany \\ ${ }^{b}$ Physikalisches Institut, Albert-Ludwigs-Universität, \\ Freiburg D-79104, Germany \\ ${ }^{c}$ Trinity College Dublin, School of Mathematics, \\ Dublin 2, Ireland \\ E-mail: harlander@physik.rwth-aachen.de, \\ mario.prausa@physik.uni-freiburg.de, usovitsj@maths.tcd.ie
}

ABSTRACT: An analytical expression for the three-loop form factors for $g g H$ and $\gamma \gamma H$ is derived for the contributions which involve massless quark loops. The result is expressed in terms of harmonic polylogarithms. It fully agrees with previously obtained kinematical expansions, and confirms a recent semi-numerical approximation which extends over the full kinematic range.

KeYwords: NLO Computations, QCD Phenomenology

ARXIV EPRINT: 1907.06957 


\section{Contents}

1 Introduction 1

2 Calculation 3

2.1 Toolchain 3

2.2 Calculation of master integrals 5

$\begin{array}{lll}3 & \text { Results } & 6\end{array}$

3.1 Results for $C_{\gamma \gamma H} \quad 6$

3.2 Results for $C_{g g H} \quad 7$

4 Conclusions 9

$\begin{array}{ll}\text { A Results for } C_{\gamma \gamma H} & 10\end{array}$

B Results for $C_{g g H} \quad 13$

$\begin{array}{ll}\text { C Ancillary file } & 18\end{array}$

\section{Introduction}

The study of the Higgs boson is one of the most promising ways to search for physics beyond the Standard Model (SM). A necessary precondition for this to be successful is the precise understanding of the relevant SM predictions. One of the most important quantities in this respect is the cross section for Higgs production in gluon fusion. In fact, significant theoretical efforts have been made to pin down its SM value, and to estimate the associated uncertainties (see ref. [1] for a recent review). One source of uncertainties is the fact that, up to now, QCD corrections to the Higgs cross section beyond next-to-leading order (NLO) are based on the approximation of an infinitely heavy quark mediating the gluon-Higgs coupling. For the top-quark contribution, which by far dominates the total cross section at the Large Hadron Collider (LHC), comparison of this limit to the full result at NLO shows agreement at the sub-\% level for a Higgs mass of $M_{\mathrm{H}}=125 \mathrm{GeV}$, providing confidence in using this approximation also at higher orders of perturbation theory $[2,3]$. In fact, an explicit calculation of sub-leading terms in $1 / m_{\mathrm{t}}$ at next-to-next-to-leading order (NNLO), combined with the high-energy limit of the cross section, further justifies this procedure [4-7]. Nevertheless, the lack of the exact top mass dependence still requires one to associate with it an uncertainty on the total cross section of the order of $1 \%$. It is thus a non-negligible contribution to the overall uncertainty of about 5\%, which also includes uncertainties induced by parton density functions (PDFs) and $\alpha_{s}$, for example (see refs. $[8,9]$ ).

A related uncertainty arises from the bottom-quark induced Higgs-gluon coupling. While suppressed by the bottom Yukawa coupling, its effect on the leading order (LO) cross 
section is still a reduction by about $6 \%$. Since the numerical value of the bottom-quark mass prohibits the analogous approximation as for the top quark, QCD corrections to the bottom-quark induced $g g H$ amplitude are known only through NLO, without significant progress since their original calculation of more than 25 years ago [3]. Serious attempts to capture the dominant logarithmic contributions of the form $\ln m_{\mathrm{b}} / M_{\mathrm{H}}$ to higher orders in perturbation theory have been presented only recently [10]. Thus, also for this source, the LHC Higgs Cross Section Working Group assigned an uncertainty of roughly another 1\% to the total gluon fusion Higgs cross section [8].

The total cross section at NNLO requires the inclusion of three-loop virtual corrections to the $g g H$ amplitude (the "Higgs-gluon form factor"), two-loop corrections to singlereal emission, and the one-loop double-real emission contributions which occur for the first time at this order. The real-emission contributions are sufficient if one aims for Higgs boson production at non-zero transverse momenta $p_{\perp}$. In this case, top-mass effects have been addressed by several groups recently [11-14]. After estimates based on $1 / m_{\mathrm{t}}$ expansions of the cross section which indicated a break-down of this approximation for $p_{\perp} \gtrsim 150 \mathrm{GeV}[15,16]$, it came as a surprise to find the K-factor of the exact calculation to be fairly independent of $p_{\perp}[12,13]$. This provides yet another indication that also for the total cross section, the QCD corrections are well described by their heavy-top limit. Also bottom-quark mass effects have been considered for finite $p_{\perp}[17,18]$ at this order of perturbation theory.

Concerning the virtual corrections, it took about ten years before the original numerical two-loop result for the Higgs-gluon form factor of ref. [3], contributing to the total cross section at NLO, was expressed in closed analytic form using harmonic polylogarithms [1921]. The analytic result for the $\gamma \gamma H$ amplitude had been obtained one year earlier [22].

For the three-loop form factor, only approximate results are available up to now, most notably through expansions in the heavy-quark limit [23-26]. While this expansion is expected to work very well for on-shell Higgs production mediated by a top-quark loops, it will break down for the bottom-mediated contribution, or in cases where the Higgs is produced as a virtual intermediate particle, for example in off-shell or double-Higgs production. Knowledge of the general dependence of the three-loop Higgs-gluon form factor on the quark/Higgs mass ratio is thus very desirable.

Very recently, the expansions in $1 / m_{\mathrm{t}}$ were combined with the leading behavior of the amplitude at the top-threshold, i.e. $\hat{s} \approx 4 m_{\mathrm{t}}^{2}$ (see also ref. [27]) in order to construct Padé approximants for the three-loop $g g H$ amplitude which should be valid — within intrinsic Padé uncertainties - for general Higgs and quark masses [28].

In this paper, we provide an analytic result for a subset of the virtual three-loop corrections, namely those involving light (massless) quark loops in addition to the massive (top- or bottom) quark loop. Using integration-by-parts (IBP) identities, we reduce the occurring Feynman integrals to a set of master integrals, which we manage to solve in terms of harmonic polylogarithms. Comparing our result to ref. [28], we find full agreement for this light-fermion component within the uncertainty estimate of ref. [28]. As a byproduct of this calculation, we also obtain the three-loop $\gamma \gamma H$ form factor from which one may directly derive the exclusive photonic decay rate of the Higgs boson through NNLO. 


\section{Calculation}

The amplitude for the processes $g g H$ and $\gamma \gamma H$ can be parameterized with the momenta $q_{1,2}$ of the two external vector bosons as

$$
\begin{aligned}
& \mathcal{M}_{g g H}^{a b ; \mu \nu}= \delta^{a b}\left[\left(q_{1}^{\mu} q_{1}^{\nu}+q_{2}^{\mu} q_{2}^{\nu}\right) A_{g g H}+q_{1}^{\mu} q_{2}^{\nu} B_{g g H}\right. \\
&\left.+q_{2}^{\mu} q_{1}^{\nu} C_{g g H}+\left(q_{1} \cdot q_{2}\right) g^{\mu \nu} D_{g g H}\right], \\
& \mathcal{M}_{\gamma \gamma H}^{\mu \nu}=\left(q_{1}^{\mu} q_{1}^{\nu}+q_{2}^{\mu} q_{2}^{\nu}\right) A_{\gamma \gamma H}+q_{1}^{\mu} q_{2}^{\nu} B_{\gamma \gamma H}+q_{2}^{\mu} q_{1}^{\nu} C_{\gamma \gamma H}+\left(q_{1} \cdot q_{2}\right) g^{\mu \nu} D_{\gamma \gamma H},
\end{aligned}
$$

where we already implied Bose symmetry. Here and in what follows, $a$ and $b$ denote color indices of the adjoint representation, while $\mu$ and $\nu$ are $d$-dimensional Lorentz indices. Because of the trivial color structure in eq. (2.1a) which can be projected out using $\left(\delta_{a b} / N_{A}\right) \mathcal{M}_{g g H}^{a b ; \mu \nu}$, where $N_{A}$ is the number of gauge generators, we ignore the color structure in the following and focus only on the Lorentz structure of the amplitudes. For both amplitudes the Ward identity ${ }^{1}$

$$
q_{1 \mu} \epsilon_{j, \nu}\left(q_{2}\right) \mathcal{M}^{\mu \nu}=0
$$

yields a constraint

$$
D=-C
$$

In case of photons in the external state the even stronger Ward identity $q_{1 \mu} \mathcal{M}_{\gamma \gamma H}^{\mu \nu}=0$ leads in addition to a vanishing form factor $A_{\gamma \gamma H}$.

For physical quantities the only contribution stems from the form factors $C$. Therefore, the physical part of the amplitudes can be written as

$$
\begin{aligned}
\mathcal{M}_{g g H}^{a b ; \mu \nu} & =\delta^{a b}\left[q_{2}^{\mu} q_{1}^{\nu}-\left(q_{1} \cdot q_{2}\right) g^{\mu \nu}\right] C_{g g H} \\
\mathcal{M}_{\gamma \gamma H}^{\mu \nu} & =\left[q_{2}^{\mu} q_{1}^{\nu}-\left(q_{1} \cdot q_{2}\right) g^{\mu \nu}\right] C_{\gamma \gamma H} .
\end{aligned}
$$

Since gluons and photons do not directly couple to the Higgs boson, the Feynman diagrams contributing to the $g g H$ and $\gamma \gamma H$ amplitudes always involve at least one closed massive quark loop if higher orders in the electroweak coupling are neglected. In this paper, we address the calculation of the component of the form factors $C$ which, in addition to this massive quark loop, involve a closed loop of a light quark (assumed massless here). Since the corresponding Yukawa coupling vanishes, the Higgs boson will still only couple to the diagram via the massive quark loop.

In section 2.1 we describe the toolchain used to express the contribution from light quarks to the form factors $C$ in terms of master integrals. In section 2.2 the method for the calculation of the master integrals is explained.

\subsection{Toolchain}

For the calculation of the light-quark contribution to the $g g H$ and $\gamma \gamma H$ form factors it is required to evaluate the Feynman diagrams in figure 1. These Feynman diagrams are generated in a first step using the tool qgraf [29]. After the insertion of Feynman rules in

\footnotetext{
${ }^{1}$ In statements valid for both amplitudes we neglect the specification $g g H$ or $\gamma \gamma H$ in the notation.
} 


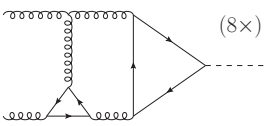

(a)

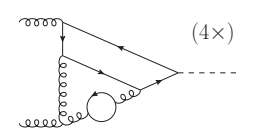

(f)

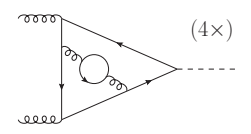

(k)

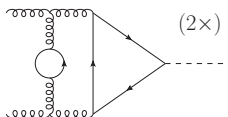

(b)

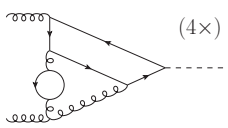

(g)

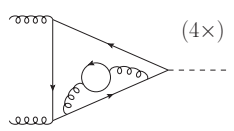

(1)

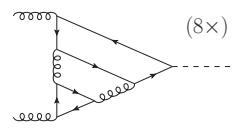

(c)

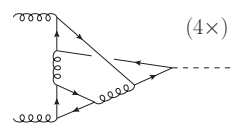

(h)

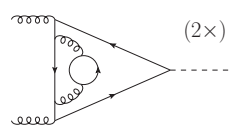

(m)

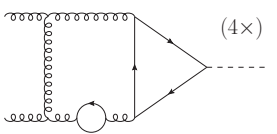

(d)

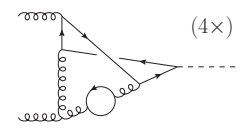

(i)

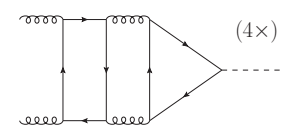

(n)

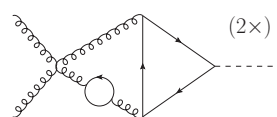

(e)

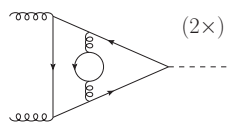

(j)

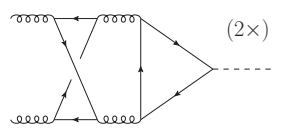

(o)

Figure 1. Feynman diagrams for $g g H$ contributing to the light-quark contribution. Diagrams with reversed fermion flows or swapped external vector bosons are not shown but indicated by the multiplicity in the top right corner of each diagram. Only diagrams $(j)-(o)$ contribute to the light-quark terms of $\gamma \gamma H$ with the external gluons replaced by photons.

$R_{\xi}$-gauge with the help of q2e $[30,31]$ the diagrams are mapped to a set of seven topologies via $\exp [30,31]$.

A custom code for the computer algebra system FORM [32] was written in order to further process the output of exp. We use the projector

$$
\mathcal{P}_{\mu \nu}=\frac{\left(q_{1} \cdot q_{2}\right) g_{\mu \nu}-q_{2 \mu} q_{1 \nu}}{\left(q_{1} \cdot q_{2}\right)^{2}(2-d)}
$$

to project out the form factor $C$ which already implies the validity of the Ward identity in eq. (2.3). Moreover, we use

$$
\begin{aligned}
& \mathcal{P}_{\mu \nu}^{(A)}=\frac{q_{1 \mu} q_{1 \nu}}{\left(q_{1} \cdot q_{2}\right)^{2}}, \\
& \mathcal{P}_{\mu \nu}^{(B)}=\frac{(1-d) q_{1 \nu} q_{2 \mu}-q_{1 \mu} q_{2 \nu}+\left(q_{1} \cdot q_{2}\right) g_{\mu \nu}}{\left(q_{1} \cdot q_{2}\right)^{2}(2-d)}, \\
& \mathcal{P}_{\mu \nu}^{(C)}=\frac{-q_{1 \nu} q_{2 \mu}+(1-d) q_{1 \mu} q_{2 \nu}+\left(q_{1} \cdot q_{2}\right) g_{\mu \nu}}{\left(q_{1} \cdot q_{2}\right)^{2}(2-d)}, \\
& \mathcal{P}_{\mu \nu}^{(D)}=\frac{q_{1 \nu} q_{2 \mu}+q_{1 \mu} q_{2 \nu}-\left(q_{1} \cdot q_{2}\right) g_{\mu \nu}}{\left(q_{1} \cdot q_{2}\right)^{2}(2-d)}
\end{aligned}
$$

to project out all the form factors in eq. (2.1) in order to check our calculational setup by explicitly verifying the validity of the Ward identities, see eq. (2.3) and below. The color factor of each diagram is determined via the FORM package color [33].

After projecting out the form factors, the results can be expressed in terms of scalar Feynman integrals, which are subsequently reduced to 45 master integrals using integration- 
by-parts identities [34,35] and the Laporta algorithm [36], implemented in the computer program Kira ${ }^{2}[37,38]$.

After the reduction to master integrals the dependence on the gauge parameter $\xi$ drops out and the validity of eq. (2.3) as well as $A_{\gamma \gamma H}=0$ is confirmed.

\subsection{Calculation of master integrals}

A very successful technique for the evaluation of two-scale Feynman integrals is based on the method of differential equations [39-42]. The solution of the resulting coupled system of differential equations simplifies significantly if it can be written in the canonical form proposed in ref. [43], where the right-hand side of the system is proportional to $\epsilon=(4-d) / 2$. An algorithm to compute a basis transformation to such a canonical form was presented by Lee in ref. [44]. We utilize its implementation in the computer program epsilon [45] in order to evaluate the relevant master integrals.

The class of transformations Lee's algorithm is able to find is restricted to be rational in the kinematic variable. Hence, a proper choice for the kinematic variable is inevitable to obtain a canonical form. For our purposes, an appropriate variable is

$$
x=\frac{\sqrt{1-1 / \tau}-1}{\sqrt{1-1 / \tau}+1},
$$

where $\tau=M_{\mathrm{H}}^{2} /\left(4 m_{\mathrm{q}}^{2}\right)+i 0$, with the mass $M_{\mathrm{H}}$ of the Higgs boson and the mass $m_{\mathrm{q}}$ of the massive quark.

Ordering the master integrals by the number of lines in their topology yields a blocktriangular structure of the system of differential equations. For most applications it is sufficient to transform only the on-diagonal blocks into the previously described canonical form. The differential equations for master integrals of a certain block $\vec{f}(x, \epsilon)$ can then be written as

$$
\frac{\partial}{\partial x} \vec{f}(x, \epsilon)=\epsilon M(x) \vec{f}(x, \epsilon)+B(x, \epsilon) \vec{g}(x, \epsilon),
$$

where $\vec{g}(x, \epsilon)$ consists of already solved master integrals of a lower topology, and $M(x)$ is fuchsian, i.e. it possesses only simple poles in $x$. For the master integrals entering the light-quark contributions, these poles lie at $x=-1,0,1$. The homogeneous part of (2.8) can be solved using an evolution operator $U\left(x, x_{0} ; \epsilon\right)$ which fulfills

$$
\frac{\partial}{\partial x} U\left(x, x_{0} ; \epsilon\right)=\epsilon M(x) U\left(x, x_{0} ; \epsilon\right) ; \quad U\left(x_{0}, x_{0} ; \epsilon\right)=\mathbb{1},
$$

via iterated integrations in terms of multiple polylogarithms. This evolution operator allows expressing the full solution as

$$
\vec{f}(x, \epsilon)=\int_{x_{0}}^{x} \mathrm{~d} x^{\prime} U\left(x, x^{\prime} ; \epsilon\right) B\left(x^{\prime}, \epsilon\right) \vec{g}\left(x^{\prime}, \epsilon\right)+U\left(x, x_{0} ; \epsilon\right) \vec{f}\left(x_{0}, \epsilon\right),
$$

where $\vec{f}\left(x_{0}, \epsilon\right)$ are the boundary conditions of the master integrals at $x=x_{0}$. In order to simplify the integral in (2.10) we chose to transform the off-diagonal blocks $B(x, \epsilon)$ of the

\footnotetext{
${ }^{2}$ We note that Kira is also able to completely reduce the full form factors $C$ (including the $n_{l}^{0}$-terms) in Feynman gauge to 403 master integrals.
} 
system of differential equations into fuchsian form via epsilon. Doing that ensures $\vec{f}(x, \epsilon)$ to be a linear combination of multiple polylogarithms without rational function prefactors in case $\vec{g}(x, \epsilon)$ is of this form as well.

The boundary conditions $\vec{f}\left(x_{0}, \epsilon\right)$ are calculated as an asymptotic expansion around $x_{0}=1$, which corresponds to a limit where the quark mass $m_{\mathrm{q}}$ is large compared to the Higgs mass $M_{\mathrm{H}}$. For this purpose, we expand the master integrals by subgraphs [46-49] as it is implemented in the computer program exp [30,31].

Via the method described in this section we were able to solve not only the 45 master integrals relevant for the light-quark contributions, but in total 202 master integrals for the full amplitudes (including $n_{l}^{0}$ terms). The master integrals entering the light-quark contributions were cross checked against numerical results obtained by the package FIESTA [50].

\section{Results}

In this section, we define the parameterization of our results, which we have evaluated for a general gauge group with fundamental and adjoint quadratic Casimir eigenvalues $C_{F}$ and $C_{A}$, and fundamental trace normalization $T_{F}$. For QCD, it is $C_{F}=4 / 3, C_{A}=3$, and $T_{F}=1 / 2$. The actual analytic expressions are deferred to the appendix for the sake of readability of the main text. In this section, we restrict ourselves to a numerical presentation of the results.

\subsection{Results for $C_{\gamma \gamma H}$}

The form factor $C_{\gamma \gamma H}$ is presented as a perturbative series in the strong coupling constant $\alpha_{s}$, renormalized in $n_{l}$-flavor QCD in the $\overline{\mathrm{MS}}$ scheme:

$$
C_{\gamma \gamma H}=\frac{1}{v} \frac{\alpha}{\pi}\left[C_{\gamma \gamma H}^{(0)}+\frac{\alpha_{s}}{\pi} C_{\gamma \gamma H}^{(1)}+\left(\frac{\alpha_{s}}{\pi}\right)^{2} C_{\gamma \gamma H}^{(2)}+\mathcal{O}\left(\alpha_{s}^{3}\right)\right]
$$

where $v$ denotes the vacuum expectation value and $\alpha$ the electromagnetic coupling constant. In order to fix the notation we provide the one-loop result as

$$
C_{\gamma \gamma H}^{(0)}=\frac{C_{A} Q_{\mathrm{q}}^{2}}{T_{F}}\left[-\frac{2 x}{(1-x)^{2}}+\frac{x(1+x)^{2}}{(1-x)^{4}} H_{0,0}\right],
$$

with the electric charge $Q_{\mathrm{q}}$ of the massive quark and $H_{0,0}=\ln ^{2}(x) / 2$. The three-loop result can be parameterized via

$$
C_{\gamma \gamma H}^{(2)}=C_{\gamma \gamma H}^{(2,0)}+C_{A} C_{F} Q_{\mathrm{q}}^{2} n_{l} \mathcal{C}_{\gamma \gamma H}^{(\text {non-sing })}+C_{A} C_{F} \sum_{j=1}^{n_{l}} Q_{j}^{2} \mathcal{C}_{\gamma \gamma H}^{(\text {sing })},
$$

where $Q_{j}$ are the electric charges of the $n_{l}$ light quarks. The term $C_{\gamma \gamma H}^{(2,0)}$ denotes the part of the amplitude without a massless quark loop, which we have not computed. The contribution stemming from a massless quark loop is split into a non-singlet part $\mathcal{C}_{\gamma \gamma H}^{\text {(non-sing) }}$ and a singlet part $\mathcal{C}_{\gamma \gamma H}^{(\mathrm{sing})}$. The singlet part contains the contributions from the diagrams figures $1(\mathrm{n})-1(\mathrm{o})$, where the external photons couple to the light quark loop. 


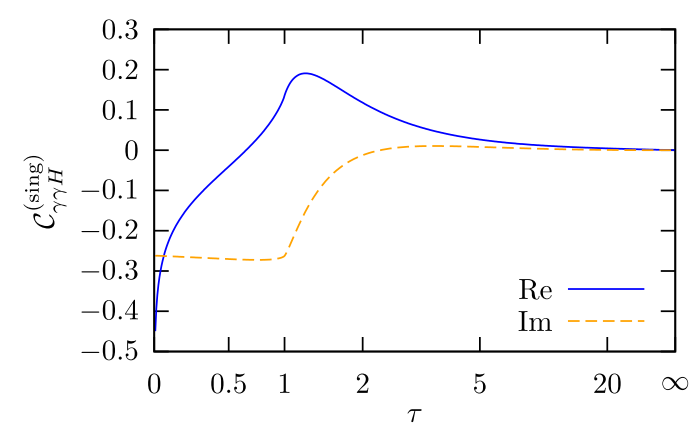

(a)

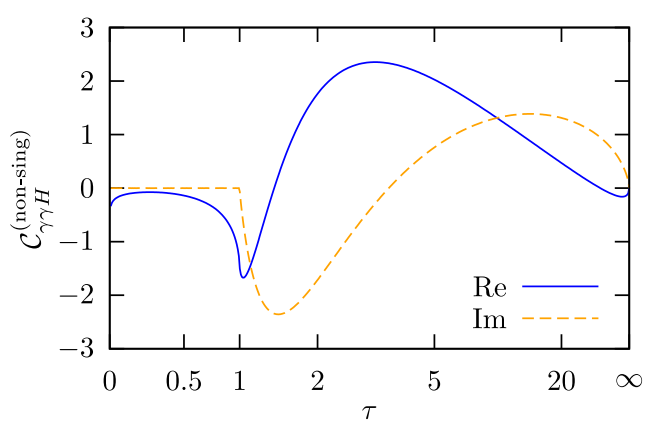

(b)

Figure 2. Singlet and non-singlet part of $C_{\gamma \gamma H}^{(2)}$ with the quark mass renormalized in the on-shell scheme and $\mu^{2}=M_{\mathrm{H}}^{2}$.

The explicit results for $\mathcal{C}_{\gamma \gamma H}^{\text {(non-sing) }}$ and $\mathcal{C}_{\gamma \gamma H}^{\text {(sing) }}$ are presented along with the two-loop results in appendix A, both for an on-shell and an $\overline{\mathrm{MS}}$-renormalized massive quark mass. In addition, we provide them in electronic form in an ancillary file, see appendix C. The $\overline{\mathrm{MS}}$-renormalized result, expanded around $x=1$ up to the order $\mathcal{O}\left((1-x)^{40}\right)$, agrees with ref. [24].

Figure 2 shows the real and imaginary part of the three-loop amplitude, separately for the singlet and the non-singlet component.

\subsection{Results for $C_{g g H}$}

The form factor $C_{g g H}$ can also be written as a series in $\alpha_{s}$,

$$
C_{g g H}=\frac{1}{v} \frac{\alpha_{s}}{\pi}\left[C_{g g H}^{(0)}+\frac{\alpha_{s}}{\pi} C_{g g H}^{(1)}+\left(\frac{\alpha_{s}}{\pi}\right)^{2} C_{g g H}^{(2)}+\mathcal{O}\left(\alpha_{s}^{3}\right)\right] .
$$

We provide again the one-loop result to fix the notation,

$$
C_{g g H}^{(0)}=T_{F}\left[-\frac{4 x}{(1-x)^{2}}+\frac{2 x(1+x)^{2}}{(1-x)^{4}} H_{0,0}\right] .
$$

In contrast to the $C_{\gamma \gamma H}$ form factor, the purely virtual $g g H$ result is not finite after the ultraviolet renormalization procedure. This is due to infrared divergences which cancel against real corrections, or are absorbed into PDFs. In ref. [51] it is shown that the structure of these infrared divergences is universal and can be subtracted:

$$
\begin{aligned}
& \tilde{C}_{g g H}^{(1)}=C_{g g H}^{(1)}-\frac{1}{2} I_{g}^{(1)} C_{g g H}^{(0)}, \\
& \tilde{C}_{g g H}^{(2)}=C_{g g H}^{(2)}-\frac{1}{2} I_{g}^{(1)} C_{g g H}^{(1)}-\frac{1}{4} I_{g}^{(2)} C_{g g H}^{(0)} .
\end{aligned}
$$

The factors $I_{g}^{(1)}$ and $I_{g}^{(2)}$ are given by [51, 52]

$$
\begin{aligned}
& I_{g}^{(1)} \equiv I_{g}^{(1)}(\epsilon)=-\left(-\frac{\mu^{2}}{M_{\mathrm{H}}^{2}}\right)^{\epsilon} \frac{e^{\epsilon \gamma_{E}}}{\Gamma(1-\epsilon)}\left[\frac{C_{A}}{\epsilon^{2}}+\frac{\beta_{0}}{\epsilon}\right], \\
& I_{g}^{(2)=-} \frac{1}{2} I_{g}^{(1)}(\epsilon)\left(I_{g}^{(1)}(\epsilon)+\frac{\beta_{0}}{\epsilon}\right)+\frac{e^{-\epsilon \gamma_{E}} \Gamma(1-2 \epsilon)}{\Gamma(1-\epsilon)}\left(\frac{\beta_{0}}{\epsilon}+K\right) I_{g}^{(1)}(2 \epsilon) \\
&+\frac{e^{\epsilon \gamma_{E}}}{\Gamma(1-\epsilon)} \frac{H_{g}}{2 \epsilon},
\end{aligned}
$$




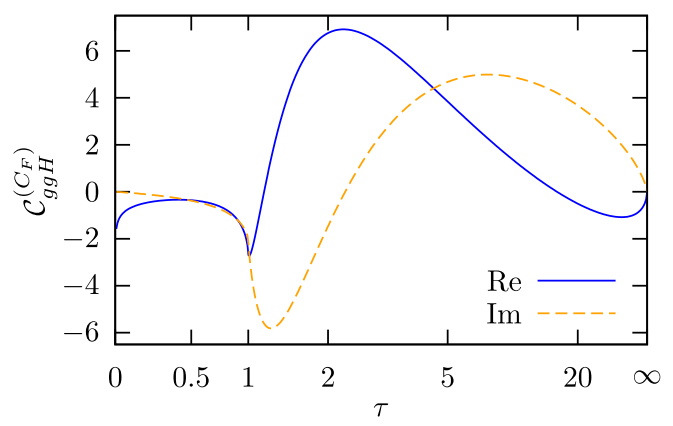

(a)

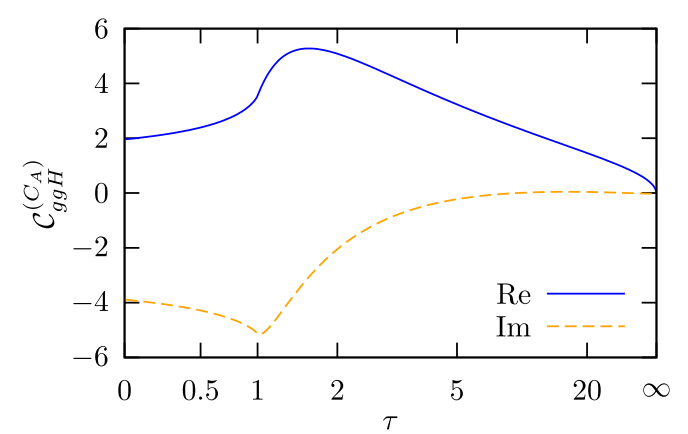

(b)

Figure 3. Contributions to $\tilde{C}_{g g H}^{(2)}$ separated by their color factors. The quark mass is renormalized in the on-shell scheme and the renormalization scale is set to $\mu^{2}=M_{\mathrm{H}}^{2}$.

and

$$
\begin{aligned}
\beta_{0} & =\frac{11}{6} C_{A}-\frac{2}{3} T_{F} n_{l} \\
K & =\left(\frac{67}{18}-\frac{\pi^{2}}{6}\right) C_{A}-\frac{10}{9} T_{F} n_{l}, \\
H_{g} & =\frac{20}{27} T_{F}^{2} n_{l}^{2}+T_{F} C_{F} n_{l}-\left(\frac{\pi^{2}}{36}+\frac{58}{27}\right) T_{F} n_{l} C_{A}+\left(\frac{\zeta_{3}}{2}+\frac{5}{12}+\frac{11 \pi^{2}}{144}\right) C_{A}^{2} .
\end{aligned}
$$

The finite three-loop terms can be parameterized as

$$
\tilde{C}_{g g H}^{(2)}=\tilde{C}_{g g H}^{(2,0)}+n_{l} \tilde{C}_{g g H}^{(2,1)}+n_{l}^{2} \tilde{C}_{g g H}^{(2,2)},
$$

where $\tilde{C}_{g g H}^{(2,0)}$ denotes the contribution of Feynman diagrams without a light quark loop. Since at the three-loop level there are no diagrams with more than two closed quark loops, the $\tilde{C}_{g g H}^{(2,2)}$ contribution originates solely from the subtraction terms of eq. (3.6b). We further decompose $\tilde{C}_{g g H}^{(2,1)}$ into its contributions to different color factors,

$$
\tilde{C}_{g g H}^{(2,1)}=T_{F}^{2} C_{F} \mathcal{C}_{g g H}^{\left(C_{F}\right)}+T_{F}^{2} C_{A} \mathcal{C}_{g g H}^{\left(C_{A}\right)} .
$$

Explicit results for $\mathcal{C}_{g g H}^{\left(C_{F}\right)}$ and $\mathcal{C}_{g g H}^{\left(C_{A}\right)}$, where the quark mass again has been renormalized both in the $\overline{\mathrm{MS}}$ and the on-shell scheme, are given along with the two-loop results in appendix $\mathrm{B}$ and in an ancillary file, see appendix $\mathrm{C}$. The result renormalized in the onshell scheme, expanded around $x=1$ up to $\mathcal{O}\left((1-x)^{12}\right)$, agrees with refs. [5, 26, 28]. Also all terms of the threshold expansion given in refs. [27, 28] could be reproduced. The real and imaginary part of the result is shown in figure 3.

Figure 4 compares these results to the semi-numerical approximation of ref. [28]. ${ }^{3}$ The latter is associated with a systematic uncertainty due to the approximation procedure, which is dominated at large $\tau$ by the absence of any input from this kinematical region into the Padé approximants. Our results indeed confirm the associated uncertainty estimate for the light-quark terms up to rather large values of $\tau$ (corresponding to large Higgs masses/virtualities or small quark masses).

\footnotetext{
${ }^{3}$ In accordance with ref. [28], we set $\mu^{2}=-M_{\mathrm{H}}^{2}$ in figure 4 .
} 


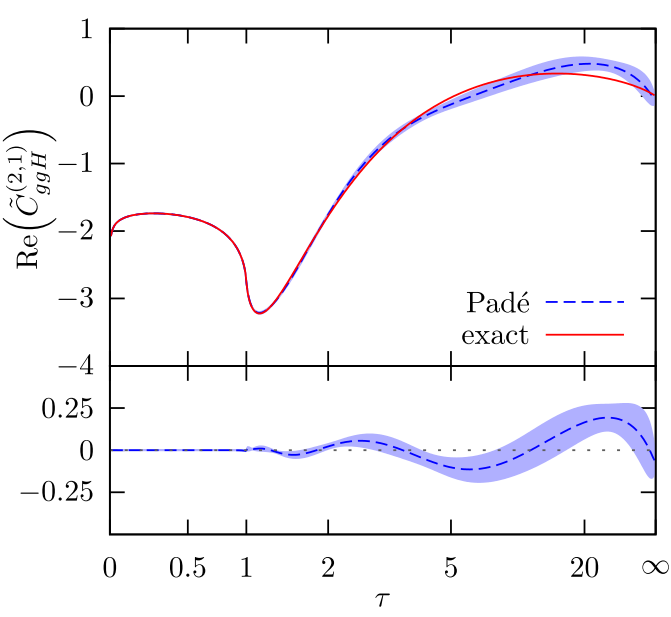

(a)

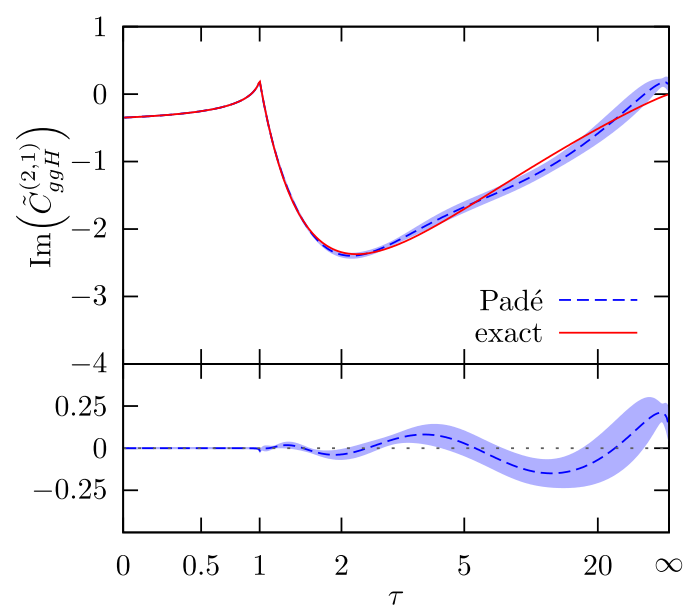

(b)

Figure 4. Real and imaginary part of $\tilde{C}_{g g H}^{(2,1)}$ with the quark mass renormalized on-shell, $\mu^{2}=$ $-M_{\mathrm{H}}^{2}$, and the color factors set to their $\mathrm{SU}(3)$ values. The red (solid) line is the result given in eqs. (3.10), (B.4), (B.5). The blue (dashed) line shows the Padé approximation found in ref. [28]; the associated uncertainty estimate is indicated by the blue shaded band. The lower panel shows the difference between the Padé approximation and our result.

\section{Conclusions}

The Higgs-gluon form factor is an essential component for the theoretical description of Higgs physics at hadron colliders. It enters the total cross section for single and double Higgs production at the LHC, for example. In QCD, it involves a massive quark loop which mediates the Higgs-gluon/photon coupling. Until recently, the three-loop $g g H$ form factor has been known only in the limit of a very heavy mediating quark. This has restricted its applicability to top-quark mediated on-shell (or not-too off-shell) single production of the SM-like Higgs boson. Even there, the lack of an exact result implied non-negligible uncertainties. In cases such as double-Higgs production, off-shell or bottom-quark mediated single-Higgs production, or the production of heavy beyond-the-SM (BSM) Higgs bosons, the expansion fails and one had to resort to the NLO result.

In this paper, we provided an analytic three-loop result for the component of the gluonHiggs form factor which, in addition to the massive quark loop, involves a closed massless quark loop. We showed that it can be computed in closed form for a general quark mass, and presented it in terms of harmonic polylogarithms. Comparison of this result to a recent semi-numerical evaluation of the full Higgs-gluon form factor shows very good agreement at the level of the estimated numerical uncertainties. As a byproduct of our calculation, we also presented the analogous component of the amplitude for the decay rate of the Higgs boson into photons.

A large portion of our technical setup is applicable also to the full form factor. However, the calculations are much more expensive than for the light-quark terms considered here. Moreover, one encounters elliptic integrals which cannot be expressed in terms of harmonic polylogarithms. A fully analytical result for the three-loop form factor therefore requires further efforts. 


\section{Acknowledgments}

We would like to thank Florian Herren and Philipp Maierhöfer for helpful discussions, and Matthias Steinhauser for useful comments on the manuscript. This work was supported by Deutsche Forschungsgemeinschaft (DFG) through the Collaborative Research Center TRR 257 "Particle Physics Phenomenology after the Higgs Discovery". J.U. received funding from the European Research Council (ERC) under the European Unions Horizon 2020 research and innovation programme under grant agreement no. 647356 (CutLoops). The authors acknowledge support by the state of Baden-Württemberg through bwHPC and the German Research Foundation (DFG) through grant no INST 39/963-1 FUGG. Parts of the computing resources were granted by RWTH Aachen University under project rwth0119. We would also like to thank Peter Uwer and his group "Phenomenology of Elementary Particle Physics beyond the Standard Model" at Humboldt-Universität zu Berlin for providing computer resources. The Feynman diagrams in this article have been drawn with JaxoDraw [53] based on Axodraw [54].

\section{A Results for $C_{\gamma \gamma H}$}

In this appendix, we provide explicit formulas for the two-loop result and the newly computed light-quark contributions to the three-loop result of the $\gamma \gamma H$ form factor, cf. eqs. (3.1), (3.3). We use $H_{\vec{a}} \equiv H(\vec{a} ; x)$ to denote harmonic polylogarithms $[55,56]$, $\zeta_{n} \equiv \sum_{j=1}^{\infty} j^{-n}$ for Riemann's zeta function, and the short-hand notation $L_{\mu} \equiv \ln \left(\mu^{2} / m_{\mathrm{q}}^{2}\right)$ with the renormalization scale $\mu$.

The two-loop result has been know for about 15 years [22]. In our notation, it reads

$$
\begin{aligned}
& C_{\gamma \gamma H}^{(1)}=\frac{C_{A} C_{F} Q_{\mathrm{q}}^{2}}{T_{F}}\{-\frac{5 x}{(1-x)^{2}}+\frac{x\left(1-14 x+x^{2}\right)}{(1-x)^{4}} \zeta_{3}-\frac{3 x(1+x)}{(1-x)^{3}} H_{0}+\frac{6 x^{2}}{(1-x)^{4}} H_{0,0} \\
&-\frac{x\left(5-6 x+5 x^{2}\right)}{(1-x)^{4}} H_{1,0,0}+\frac{x\left(3+25 x-7 x^{2}+3 x^{3}\right)}{2(1-x)^{5}} H_{0,0,0} \\
&+\frac{x(1+x)^{2}}{6(1-x)^{4}}\left[-\pi^{2} H_{0}-24 H_{0,-1,0}+6 H_{0,1,0}\right]+\frac{x(1+x)\left(1+x^{2}\right)}{60(1-x)^{5}} \\
& \times\left[-3 \pi^{4}-20 \pi^{2} H_{0,0}+240 H_{0,-1,0,0}-480 H_{0,0,-1,0}-30 H_{0,0,0,0}\right. \\
&\left.\left.+120 H_{0,0,1,0}-420 H_{0,1,0,0}-240 H_{0} \zeta_{3}\right]\right\} \\
& \bar{C}_{\gamma \gamma H}^{(1)}=C_{\gamma \gamma H}^{(1)}+\Delta C_{\gamma \gamma H}^{(1)}, \\
& \Delta C_{\gamma \gamma H}^{(1)}=\frac{C_{A} C_{F} Q_{\mathrm{q}}^{2}}{T_{F}}\left\{\frac{x}{(1-x)^{2}}\left[-4-3 L_{\mu}\right]+\frac{x(1+x)}{2(1-x)^{3}}\left[4 H_{0}+3 H_{0} L_{\mu}\right]\right. \\
&\left.+\frac{x\left(1+6 x+x^{2}\right)}{2(1-x)^{4}}\left[4 H_{0,0}+3 H_{0,0} L_{\mu}\right]\right\}
\end{aligned}
$$

where $C_{\gamma \gamma H}^{(1)}\left(\bar{C}_{\gamma \gamma H}^{(1)}\right)$ is the result for a quark mass renormalized in the on-shell $(\overline{\mathrm{MS}})$ scheme. 
At three loops, the non-singlet contribution, with the quark mass renormalized in the on-shell scheme, is given by

$$
\begin{aligned}
& \mathcal{C}_{\gamma \gamma H}^{\text {(non-sing) }}=-\frac{(5-7 x) x}{18(1-x)^{3}} \pi^{2}-\frac{x\left(1-14 x+x^{2}\right)}{3(1-x)^{4}} L_{\mu} \zeta_{3}+\frac{x\left(13+118 x+13 x^{2}\right)}{9(1-x)^{4}} \zeta_{3} \\
& +\frac{x\left(63-85 x+73 x^{2}+21 x^{3}\right)}{1080(1-x)^{5}} \pi^{4}-\frac{4 x\left(1-4 x+x^{2}\right)}{3(1-x)^{4}} H_{1} \zeta_{3}+\frac{x\left(17+10 x-x^{2}\right)}{54(1-x)^{4}} \\
& \times \pi^{2} H_{0}+\frac{2 x\left(16+19 x-20 x^{2}+13 x^{3}\right)}{9(1-x)^{5}} H_{0} \zeta_{3}-\frac{2 x^{2}}{(1-x)^{4}} H_{0,0} L_{\mu} \\
& +\frac{x\left(3-14 x-10 x^{2}\right)}{3(1-x)^{4}} H_{0,0}+\frac{x\left(47+95 x-115 x^{2}+5 x^{3}\right)}{108(1-x)^{5}} \pi^{2} H_{0,0} \\
& -\frac{2 x\left(7+29 x+7 x^{2}\right)}{9(1-x)^{4}} H_{0,1,0}-\frac{x\left(33+499 x-265 x^{2}-99 x^{3}\right)}{36(1-x)^{5}} H_{0,0,0} \\
& -\frac{x\left(3+25 x-7 x^{2}+3 x^{3}\right)}{6(1-x)^{5}} H_{0,0,0} L_{\mu}+\frac{4 x\left(11+34 x+11 x^{2}\right)}{9(1-x)^{4}} H_{0,-1,0} \\
& -\frac{2 x\left(3-2 x+3 x^{2}\right)}{3(1-x)^{4}} H_{1,1,0,0}-\frac{x\left(2+62 x+17 x^{2}+17 x^{3}\right)}{9(1-x)^{5}} H_{0,0,0,0} \\
& -\frac{x\left(47+119 x-67 x^{2}+29 x^{3}\right)}{18(1-x)^{5}} H_{0,0,1,0}+\frac{8 x(1+x)\left(2+6 x-7 x^{2}\right)}{9(1-x)^{5}} H_{0,-1,0,0} \\
& +\frac{x\left(17-42 x+17 x^{2}\right)}{6(1-x)^{4}} H_{1,0,0,0}+\frac{4 x\left(19+31 x-23 x^{2}+13 x^{3}\right)}{9(1-x)^{5}} H_{0,0,-1,0} \\
& +\frac{2 x\left(10-11 x-11 x^{2}+34 x^{3}\right)}{9(1-x)^{5}} H_{0,1,0,0}+\frac{x(1+x)}{6(1-x)^{3}}\left[23 H_{0}-16 H_{-1,0}+10 H_{1,0}\right. \\
& \left.+6 H_{0} L_{\mu}\right]+\frac{x\left(5-6 x+5 x^{2}\right)}{9(1-x)^{4}}\left[5 H_{1,0,0}+3 H_{1,0,0} L_{\mu}\right]+\frac{x}{18(1-x)^{2}}[101 \\
& \left.-\pi^{2} H_{1,0}-96 H_{1,0,-1,0}+42 H_{1,0,1,0}+30 L_{\mu}\right]+\frac{x(1+x)^{2}}{18(1-x)^{4}}\left[2 \pi^{2} H_{0,1}\right. \\
& -96 H_{0,-1,-1,0}+48 H_{0,-1,1,0}+48 H_{0,1,-1,0}-12 H_{0,1,1,0}+\pi^{2} H_{0} L_{\mu}-6 H_{0,1,0} L_{\mu} \\
& \left.+24 H_{0,-1,0} L_{\mu}\right]+\frac{x(1+x)\left(1+x^{2}\right)}{540(1-x)^{5}}\left[-18 \pi^{4} H_{-1}+14 \pi^{4} H_{0}-120 \pi^{2} H_{-1,0,0}\right. \\
& +120 \pi^{2} H_{0,0,0}+120 \pi^{2} H_{0,0,1}+1440 H_{-1,0,-1,0,0}-2880 H_{-1,0,0,-1,0}+9 \pi^{4} L_{\mu} \\
& -180 H_{-1,0,0,0,0}+720 H_{-1,0,0,1,0}-2520 H_{-1,0,1,0,0}+1440 H_{0,-1,-1,0,0}-30 \pi^{2} \zeta_{3} \\
& +1440 H_{0,-1,0,-1,0}-1800 H_{0,-1,0,0,0}-720 H_{0,-1,0,1,0}-5760 H_{0,0,-1,-1,0} \\
& +2880 H_{0,0,-1,0,0}+2880 H_{0,0,-1,1,0}+2880 H_{0,0,0,-1,0}+270 H_{0,0,0,0,0}+2520 \zeta_{5} \\
& -720 H_{0,0,0,1,0}+2880 H_{0,0,1,-1,0}-720 H_{0,0,1,0,0}-720 H_{0,0,1,1,0}+3060 H_{0,1,0,0,0}
\end{aligned}
$$




$$
\begin{aligned}
& -4320 H_{0,1,0,-1,0}+2160 H_{0,1,0,1,0}-360 H_{0,1,1,0,0}+60 \pi^{2} H_{0,0} L_{\mu}+90 H_{0,0,0,0} L_{\mu} \\
& -720 H_{0,-1,0,0} L_{\mu}+1440 H_{0,0,-1,0} L_{\mu}-360 H_{0,0,1,0} L_{\mu}+1260 H_{0,1,0,0} L_{\mu} \\
& \left.-1440 H_{-1,0} \zeta_{3}-360 H_{0,-1} \zeta_{3}+1080 H_{0,0} \zeta_{3}-1800 H_{0,1} \zeta_{3}+720 H_{0} L_{\mu} \zeta_{3}\right] \cdot(\mathrm{A} .
\end{aligned}
$$

The singlet contribution is

$$
\begin{aligned}
& \mathcal{C}_{\gamma \gamma H}^{\text {(sing) }}=-\frac{x\left(37-243 x+249 x^{2}-15 x^{3}\right)}{6(1-x)^{5}} \zeta_{3}+\frac{5 x\left(1-22 x+x^{2}\right)}{3(1-x)^{4}} \zeta_{5} \\
& +\frac{x\left(3+44 x-68 x^{2}+20 x^{3}+15 x^{4}\right)}{1080(1-x)^{6}} \pi^{4}-\frac{39 x^{2}}{2(1-x)^{3}} H_{0}-\frac{x^{2}\left(4-x+11 x^{2}\right)}{18(1-x)^{5}} \pi^{2} H_{0} \\
& +\frac{3 x}{2(1-x)^{2}}\left[2+13 H_{1}\right]+\frac{4 x\left(1+3 x+x^{2}\right)}{(1-x)^{4}} H_{1} \zeta_{3}+\frac{2 x^{2}\left(14-20 x-4 x^{2}+3 x^{3}\right)}{3(1-x)^{6}} \\
& \times H_{0} \zeta_{3}-\frac{4 x\left(1+8 x+x^{2}\right)}{3(1-x)^{4}} H_{1,0} \zeta_{3}+\frac{x^{2}(35+66 x)}{6(1-x)^{4}} H_{0,0}+\frac{x^{2}\left(2-5 x+2 x^{2}-6 x^{3}\right)}{9(1-x)^{6}} \\
& \times \pi^{2} H_{0,0}-\frac{x^{2}\left(22-145 x-7 x^{2}\right)}{6(1-x)^{5}} H_{0,0,0}-\frac{2 x\left(1-x+x^{2}\right)}{9(1-x)^{4}} \pi^{2} H_{1,0,0} \\
& +\frac{x\left(13-266 x+13 x^{2}\right)}{6(1-x)^{4}} H_{1,0,0}+\frac{2 x\left(4-22 x+25 x^{2}+7 x^{3}\right)}{3(1-x)^{5}} H_{0,-1,0} \\
& +\frac{x\left(1-3 x+9 x^{2}+21 x^{3}\right)}{3(1-x)^{5}} H_{0,0,1}-\frac{2 x\left(1+2 x-7 x^{2}\right)}{(1-x)^{5}} H_{0,1,0,0}-\frac{2 x\left(1+10 x+x^{2}\right)}{(1-x)^{4}} \\
& \times H_{1,1,0,0}+\frac{2 x^{2}(1+3 x)}{(1-x)^{5}} H_{0,0,1,0}+\frac{2 x\left(1-6 x-2 x^{2}\right)}{(1-x)^{4}} H_{1,0,0,0} \\
& +\frac{x^{2}\left(2+7 x-10 x^{2}-6 x^{3}\right)}{3(1-x)^{6}} H_{0,0,0,0}+\frac{x\left(1-10 x+x^{2}\right)}{(1-x)^{4}} H_{0,1,0,0,0}+\frac{x(1+x)}{36(1-x)^{3}} \\
& \times\left[-7 \pi^{2}+444 H_{-1,0}+156 H_{0,1}-108 H_{1,0}\right]+\frac{x(1+x)\left(11-8 x+11 x^{2}\right)}{18(1-x)^{5}}\left[\pi^{2} H_{-1}\right. \\
& \left.-12 H_{-1,-1,0}-12 H_{-1,0,1}\right]+\frac{x\left(1+x^{2}\right)}{3(1-x)^{4}}\left[12 H_{1,0,-1,0}+12 H_{1,0,0,1}+\pi^{2} \zeta_{3}\right] \\
& +\frac{4 x^{2}\left(1+11 x-11 x^{2}+6 x^{3}\right)}{3(1-x)^{6}}\left[H_{0,0,-1,0}+H_{0,0,0,1}\right] \\
& +\frac{x\left(3-4 x+16 x^{2}-4 x^{3}+3 x^{4}\right)}{9(1-x)^{6}}\left[\pi^{2} H_{0,-1}-12 H_{0,-1,-1,0}-12 H_{0,-1,0,1}\right] \\
& +\frac{x\left(1-4 x+x^{2}\right)}{270(1-x)^{4}}\left[\pi^{4} H_{1}-270 H_{0,1,0}+720 H_{1,0,0,-1,0}+720 H_{1,0,0,0,1}\right]+\frac{x(1+x)^{2}}{9(1-x)^{4}} \\
& \times\left[-3 \pi^{2} H_{1,0}+2 \pi^{2} H_{0,0,-1}+4 \pi^{2} H_{1,0,-1}-24 H_{0,0,-1,-1,0}-24 H_{0,0,-1,0,1}\right. \\
& \left.-3 H_{0,0,1,0,0}-48 H_{1,0,-1,-1,0}-48 H_{1,0,-1,0,1}-6 H_{1,0,0,0,0}\right]+\frac{2 x^{2}}{3(1-x)^{4}}\left[\pi^{2} H_{0,1,0}\right.
\end{aligned}
$$




$$
\begin{aligned}
& +2 \pi^{2} H_{1,1,0}-6 H_{1,0,1,0}-24 H_{0,1,0,-1,0}-24 H_{0,1,0,0,1}-6 H_{0,1,0,1,0}-12 H_{0,1,1,0,0} \\
& -6 H_{1,0,0,1,0}-12 H_{1,0,1,0,0}-48 H_{1,1,0,-1,0}-18 H_{1,1,0,0,0}-48 H_{1,1,0,0,1} \\
& \left.-12 H_{1,1,0,1,0}-12 H_{0,1} \zeta_{3}-24 H_{1,1,1,0,0}-24 H_{1,1} \zeta_{3}\right] .
\end{aligned}
$$

The results with an $\overline{\mathrm{MS}}$ renormalized quark mass can be written as

$$
\begin{aligned}
\overline{\mathcal{C}}_{\gamma \gamma H}^{\text {(non-sing) }} & =\mathcal{C}_{\gamma \gamma H}^{\text {(non-sing) }}+\Delta \mathcal{C}_{\gamma \gamma H}^{\text {(non-sing) }} \\
\overline{\mathcal{C}}_{\gamma \gamma H}^{(\text {sing })} & =\mathcal{C}_{\gamma \gamma H}^{\text {(sing) }}
\end{aligned}
$$

where

$$
\begin{aligned}
\Delta \mathcal{C}_{\gamma \gamma H}^{\text {(non-sing })}= & \frac{x}{24(1-x)^{2}}\left[71+8 \pi^{2}+52 L_{\mu}+12 L_{\mu}^{2}\right]+\frac{x(1+x)}{48(1-x)^{3}}\left[-71 H_{0}-8 \pi^{2} H_{0}\right. \\
& \left.-52 H_{0} L_{\mu}-12 H_{0} L_{\mu}^{2}\right]+\frac{x\left(1+6 x+x^{2}\right)}{48(1-x)^{4}}\left[-71 H_{0,0}-8 \pi^{2} H_{0,0}-52 H_{0,0} L_{\mu}\right. \\
& \left.-12 H_{0,0} L_{\mu}^{2}\right] .
\end{aligned}
$$

Note that the singlet component appears for the first time at the three-loop order and is therefore renormalization scheme independent.

\section{B Results for $C_{g g H}$}

In this appendix, we provide explicit formulas for the two-loop and the newly computed light-quark contribution to the three-loop result of the $g g H$ form factor, cf. eqs. (3.4), (3.10). The notation is the same as in appendix A.

Again, the two-loop result has been known for about 15 years [19-21]. In our notation, it reads

$$
\begin{aligned}
\tilde{C}_{g g H}^{(1)}=T_{F} C_{A}\{ & \frac{2 x(3+x)(1+3 x)}{(1-x)^{4}} \zeta_{3}+\frac{x}{3(1-x)^{2}}\left[-18-11 H_{0}-22 H_{1}-11 L_{\mu}\right] \\
- & \frac{x\left(7+38 x+7 x^{2}\right)}{3(1-x)^{4}} H_{1,0,0}+\frac{x\left(11+15 x+21 x^{2}+x^{3}\right)}{2(1-x)^{5}} H_{0,0,0}+\frac{x(1+x)^{2}}{90(1-x)^{4}} \\
& \times\left[8 \pi^{4}+90 H_{0,0}+30 \pi^{2} H_{0,0}+60 \pi^{2} H_{1,0}+330 H_{0,0,1}+330 H_{0,1,0}\right. \\
& +90 H_{0,0,0,0}+360 H_{0,0,-1,0}+720 H_{1,0,-1,0}-360 H_{1,0,0,0}+165 H_{0,0} L_{\mu} \\
& \left.\left.+540 H_{0} \zeta_{3}+1080 H_{1} \zeta_{3}\right]\right\} \\
+T_{F} C_{F}\{ & -\frac{10 x}{(1-x)^{2}}+\frac{2 x\left(1-14 x+x^{2}\right)}{(1-x)^{4}} \zeta_{3}-\frac{6 x(1+x)}{(1-x)^{3}} H_{0}+\frac{12 x^{2}}{(1-x)^{4}} H_{0,0} \\
& -\frac{2 x\left(5-6 x+5 x^{2}\right)}{(1-x)^{4}} H_{1,0,0}+\frac{x\left(3+25 x-7 x^{2}+3 x^{3}\right)}{(1-x)^{5}} H_{0,0,0}
\end{aligned}
$$




$$
\begin{gathered}
+\frac{x(1+x)^{2}}{3(1-x)^{4}}\left[-\pi^{2} H_{0}-24 H_{0,-1,0}+6 H_{0,1,0}\right]+\frac{x(1+x)\left(1+x^{2}\right)}{30(1-x)^{5}}\left[-3 \pi^{4}\right. \\
-20 \pi^{2} H_{0,0}+240 H_{0,-1,0,0}-480 H_{0,0,-1,0}-30 H_{0,0,0,0}+120 H_{0,0,1,0} \\
\left.\left.\quad-420 H_{0,1,0,0}-240 H_{0} \zeta_{3}\right]\right\} \\
+n_{l} T_{F}^{2}\left\{\frac{4 x}{3(1-x)^{2}}\left[H_{0}+2 H_{1}+L_{\mu}\right]+\frac{2 x(1+x)^{2}}{3(1-x)^{4}}\left[-3 H_{0,0,0}-2 H_{0,0,1}-2 H_{0,1,0}\right.\right. \\
\tilde{\bar{C}}_{g g H}^{(1)}=\tilde{C}_{g g H}^{(1)}+\Delta \tilde{C}_{g g H}^{(1)}, \\
\Delta \tilde{C}_{g g H}^{(1)}=T_{F} C_{F}\left\{\frac{2 x}{(1-x)^{2}}\left[-4-3 L_{\mu}\right]+\frac{x(1+x)}{(1-x)^{3}}\left[4 H_{0}+3 H_{0} L_{\mu}\right]\right. \\
\left.+\frac{x\left(1+6 x+x^{2}\right)}{(1-x)^{4}}\left[4 H_{0,0}+3 H_{0,0} L_{\mu}\right]\right\}
\end{gathered}
$$

where again the symbols with (without) a bar on top denote the results for an $\overline{\mathrm{MS}}$ (on-shell) renormalized quark mass.

The contribution to the three-loop $g g H$ form factor proportional to the color factor $C_{F}$, cf. eq. (3.10), is given by

$$
\begin{aligned}
\mathcal{C}_{g g H}^{\left(C_{F}\right)}= & -\frac{(17-7 x) x}{18(1-x)^{3}} \pi^{2}-\frac{4 x\left(1-14 x+x^{2}\right)}{3(1-x)^{4}} L_{\mu} \zeta_{3}-\frac{x\left(85-939 x+957 x^{2}-19 x^{3}\right)}{9(1-x)^{5}} \zeta_{3} \\
& +\frac{2(1-3 x) x\left(19-44 x-3 x^{2}\right)}{3(1-x)^{5}} \zeta_{5}+\frac{(5-7 x) x\left(1+x^{2}\right)}{9(1-x)^{5}} \pi^{2} \zeta_{3} \\
& +\frac{x\left(33-52 x+45 x^{2}-16 x^{3}-3 x^{4}\right)}{270(1-x)^{6}} \pi^{4}+\frac{x}{9(1-x)^{2}}\left[155+411 H_{1}+60 L_{\mu}\right] \\
& +\frac{(33-104 x) x}{3(1-x)^{3}} H_{0}+\frac{4 x\left(3+40 x+3 x^{2}\right)}{3(1-x)^{4}} H_{1} \zeta_{3}+\frac{x\left(17-19 x-8 x^{2}-32 x^{3}\right)}{27(1-x)^{5}} \pi^{2} H_{0} \\
& +\frac{2 x\left(5+2 x+7 x^{2}+4 x^{3}\right)}{135(1-x)^{5}} \pi^{4} H_{1}+\frac{2 x\left(29+138 x-288 x^{2}+90 x^{3}-11 x^{4}\right)}{9(1-x)^{6}} H_{0} \zeta_{3} \\
& -\frac{x\left(5+6 x+5 x^{2}\right)}{9(1-x)^{4}} \pi^{2} H_{1,0}-\frac{4 x\left(1+13 x-11 x^{2}+x^{3}\right)}{3(1-x)^{5}} H_{0,1} \zeta_{3} \\
& +\frac{x\left(18+7 x+34 x^{2}\right)}{3(1-x)^{4}} H_{0,0}+\frac{8 x\left(1-5 x+9 x^{2}+3 x^{3}\right)}{3(1-x)^{5}} H_{1,0} \zeta_{3} \\
& +\frac{x\left(59+72 x-294 x^{2}+144 x^{3}-65 x^{4}\right)}{54(1-x)^{6}} \pi^{2} H_{0,0}+\frac{2 x\left(3-4 x+16 x^{2}-4 x^{3}+3 x^{4}\right)}{9(1-x)^{6}}
\end{aligned}
$$




$$
\begin{aligned}
& \times \pi^{2} H_{0,-1}-\frac{2 x\left(23+58 x+23 x^{2}\right)}{9(1-x)^{4}} H_{0,1,0}-\frac{x\left(33+847 x-1351 x^{2}-141 x^{3}\right)}{18(1-x)^{5}} H_{0,0,0} \\
& -\frac{2 x\left(3+25 x-7 x^{2}+3 x^{3}\right)}{3(1-x)^{5}} H_{0,0,0} L_{\mu}+\frac{4 x^{2}\left(3-x+2 x^{2}\right)}{9(1-x)^{5}} \pi^{2} H_{1,0,0} \\
& +\frac{4 x\left(5-6 x+5 x^{2}\right)}{3(1-x)^{4}} H_{1,0,0} L_{\mu}+\frac{x\left(89-930 x+89 x^{2}\right)}{9(1-x)^{4}} H_{1,0,0} \\
& +\frac{4 x\left(34-20 x+29 x^{2}-x^{3}\right)}{9(1-x)^{5}} H_{0,-1,0}+\frac{2 x\left(1-15 x+21 x^{2}+21 x^{3}\right)}{3(1-x)^{5}} H_{0,0,1} \\
& -\frac{x\left(77+245 x-229 x^{2}+35 x^{3}\right)}{9(1-x)^{5}} H_{0,0,1,0}-\frac{2 x\left(3+18 x-76 x^{2}+54 x^{3}-27 x^{4}\right)}{3(1-x)^{6}} \\
& \times H_{0,0,0,1}-\frac{2 x\left(20+186 x-258 x^{2}+90 x^{3}-17 x^{4}\right)}{9(1-x)^{6}} H_{0,0,0,0} \\
& -\frac{8 x\left(1-4 x+20 x^{2}-4 x^{3}+x^{4}\right)}{3(1-x)^{6}} H_{0,-1,0,1}-\frac{8 x\left(7-4 x+8 x^{2}-4 x^{3}+7 x^{4}\right)}{3(1-x)^{6}} \\
& \times H_{0,-1,-1,0}+\frac{16 x(1+x)\left(5+6 x-10 x^{2}\right)}{9(1-x)^{5}} H_{0,-1,0,0}+\frac{16 x\left(1-10 x+x^{2}\right)}{3(1-x)^{4}} H_{1,1,0,0} \\
& +\frac{8 x\left(1+12 x+x^{2}\right)}{3(1-x)^{4}} H_{1,0,-1,0}+\frac{2 x\left(5-14 x+5 x^{2}\right)}{(1-x)^{4}} H_{1,0,1,0}+\frac{4 x\left(11-6 x+11 x^{2}\right)}{3(1-x)^{4}} \\
& \times H_{1,0,0,1}+\frac{x\left(53-259 x+187 x^{2}-29 x^{3}\right)}{3(1-x)^{5}} H_{1,0,0,0}+\frac{4 x\left(1-86 x+82 x^{2}+25 x^{3}\right)}{9(1-x)^{5}} \\
& \times H_{0,1,0,0}+\frac{8 x\left(25+15 x-33 x^{2}+3 x^{3}+11 x^{4}\right)}{9(1-x)^{6}} H_{0,0,-1,0}-\frac{2 x(1+x)\left(1-3 x^{2}\right)}{3(1-x)^{5}} \\
& \times H_{1,0,0,0,0}-\frac{16 x(1+x)\left(5+3 x^{2}\right)}{3(1-x)^{5}} H_{0,0,-1,-1,0}-\frac{16 x\left(1+7 x-5 x^{2}+x^{3}\right)}{3(1-x)^{5}} H_{0,1,0,-1,0} \\
& +\frac{16 x\left(1-4 x+x^{2}\right)}{3(1-x)^{4}} H_{1,0,0,0,1}+\frac{16 x(1+x)\left(1+3 x^{2}\right)}{3(1-x)^{5}} H_{0,0,-1,0,1} \\
& +\frac{4 x(1+x)\left(3+4 x^{2}\right)}{3(1-x)^{5}} H_{0,0,1,0,0}+\frac{16 x\left(3-3 x+7 x^{2}+x^{3}\right)}{3(1-x)^{5}} H_{1,0,0,-1,0} \\
& +\frac{4 x\left(7+x+12 x^{2}+6 x^{3}\right)}{(1-x)^{5}} H_{0,1,0,0,0}+\frac{4 x\left(7-5 x+19 x^{2}+7 x^{3}\right)}{3(1-x)^{5}} H_{1,0,1,0,0} \\
& +\frac{4 x\left(7-17 x+31 x^{2}+7 x^{3}\right)}{3(1-x)^{5}} H_{0,1,0,0,1}+\frac{4 x\left(11+5 x+17 x^{2}+11 x^{3}\right)}{3(1-x)^{5}} H_{0,1,0,1,0} \\
& +\frac{4 x\left(13+x+25 x^{2}+13 x^{3}\right)}{3(1-x)^{5}} H_{0,1,1,0,0}+\frac{2 x(1+x)}{3(1-x)^{3}}\left[29 H_{-1,0}+19 H_{0,1}+2 H_{1,0}\right. \\
& \left.+6 H_{0} L_{\mu}\right]+\frac{x(1+x)\left(11-8 x+11 x^{2}\right)}{9(1-x)^{5}}\left[\pi^{2} H_{-1}-12 H_{-1,-1,0}-12 H_{-1,0,1}\right]
\end{aligned}
$$




$$
\begin{aligned}
+ & \frac{4 x\left(1+4 x-2 x^{2}+x^{3}\right)}{9(1-x)^{5}}\left[\pi^{2} H_{0,1,0}-6 H_{1,0,0,1,0}\right]+\frac{8 x^{2}}{3(1-x)^{4}}\left[\pi^{2} H_{1,1,0}-9 H_{1,1,0,0,0}\right. \\
& \left.-24 H_{1,1,0,-1,0}-24 H_{1,1,0,0,1}-6 H_{1,1,0,1,0}-12 H_{1,1,1,0,0}-3 H_{0,0} L_{\mu}-12 H_{1,1} \zeta_{3}\right] \\
+ & \frac{2 x(1+x)^{2}}{9(1-x)^{4}}\left[2 \pi^{2} H_{0,1}+2 \pi^{2} H_{0,0,-1}+4 \pi^{2} H_{1,0,-1}+48 H_{0,-1,1,0}+48 H_{0,1,-1,0}\right. \\
& -6 H_{0,1,0,1}-18 H_{0,1,1,0}-48 H_{1,0,-1,-1,0}-48 H_{1,0,-1,0,1}+\pi^{2} H_{0} L_{\mu}+24 H_{0,-1,0} L_{\mu} \\
& \left.-6 H_{0,1,0} L_{\mu}\right]+\frac{x(1+x)\left(1+x^{2}\right)}{270(1-x)^{5}}\left[-18 \pi^{4} H_{-1}+23 \pi^{4} H_{0}-120 \pi^{2} H_{-1,0,0}\right. \\
& +300 \pi^{2} H_{0,0,0}+240 \pi^{2} H_{0,0,1}+1440 H_{-1,0,-1,0,0}-2880 H_{-1,0,0,-1,0}+720 H_{0,0,0,0,0} \\
& -180 H_{-1,0,0,0,0}+720 H_{-1,0,0,1,0}-2520 H_{-1,0,1,0,0}+1440 H_{0,-1,-1,0,0}+18 \pi^{4} L_{\mu} \\
& +1440 H_{0,-1,0,-1,0}-3960 H_{0,-1,0,0,0}-1440 H_{0,-1,0,0,1}-2160 H_{0,-1,0,1,0} \\
& -1440 H_{0,-1,1,0,0}+4320 H_{0,0,-1,0,0}+5760 H_{0,0,-1,1,0}+7200 H_{0,0,0,-1,0} \\
& +180 H_{0,0,0,0,1}-1620 H_{0,0,0,1,0}+5760 H_{0,0,1,-1,0}-720 H_{0,0,1,0,1}-2160 H_{0,0,1,1,0} \\
& -1440 H_{0,1,-1,0,0}-1440 H_{1,0,-1,0,0}+120 \pi^{2} H_{0,0} L_{\mu}-1440 H_{0,-1,0,0} L_{\mu} \\
& +2880 H_{0,0,-1,0} L_{\mu}+180 H_{0,0,0,0} L_{\mu}-720 H_{0,0,1,0} L_{\mu}+2520 H_{0,1,0,0} L_{\mu} \\
& \left.-1440 H_{-1,0} \zeta_{3}-360 H_{0,-1} \zeta_{3}+2520 H_{0,0} \zeta_{3}+1440 H_{0} L_{\mu} \zeta_{3}\right]
\end{aligned}
$$

where the quark mass has been renormalized in the on-shell scheme. The contribution proportional to the color factor $C_{A}$ is given by

$$
\begin{aligned}
\mathcal{C}_{g g H}^{\left(C_{A}\right)}= & -\frac{4 x(3+x)(1+3 x)}{3(1-x)^{4}} L_{\mu} \zeta_{3}-\frac{x\left(79+193 x-202 x^{2}-112 x^{3}\right)}{9(1-x)^{5}} \zeta_{3} \\
& -\frac{x\left(283-708 x+460 x^{2}-420 x^{3}+427 x^{4}\right)}{3240(1-x)^{6}} \pi^{4}+\frac{(17-77 x) x}{216(1-x)^{3}} \pi^{2} \\
& -\frac{x\left(1-27 x+21 x^{2}-23 x^{3}\right)}{36(1-x)^{5}} \pi^{2} H_{0}-\frac{x\left(25-8 x-84 x^{2}+16 x^{3}+37 x^{4}\right)}{3(1-x)^{6}} H_{0} \zeta_{3} \\
& +\frac{(422-485 x) x}{54(1-x)^{3}} H_{0}-\frac{x\left(25+38 x+25 x^{2}\right)}{27(1-x)^{4}} \pi^{2} H_{1,0} \\
& -\frac{x\left(127-96 x-686 x^{2}+96 x^{3}+223 x^{4}\right)}{432(1-x)^{6}} \pi^{2} H_{0,0}+\frac{(85-91 x) x}{9(1-x)^{3}} H_{1,0} \\
& +\frac{(97-79 x) x}{9(1-x)^{3}} H_{0,1}+\frac{x(1+x)}{(1-x)^{3}} H_{-1,0}+\frac{x\left(1145-4208 x+1253 x^{2}\right)}{324(1-x)^{4}} H_{0,0} \\
& -\frac{2 x\left(74+139 x+74 x^{2}\right)}{27(1-x)^{4}} H_{0,1,0}-\frac{2 x\left(65+191 x-164 x^{2}+34 x^{3}\right)}{27(1-x)^{5}} H_{0,0,1} \\
& -\frac{x\left(130+306 x+225 x^{2}+113 x^{3}\right)}{18(1-x)^{5}} H_{0,0,0}+\frac{x\left(541+854 x+541 x^{2}\right)}{54(1-x)^{4}} H_{1,0,0}
\end{aligned}
$$




$$
\begin{aligned}
& +\frac{2 x\left(1-13 x+10 x^{2}-12 x^{3}\right)}{3(1-x)^{5}} H_{0,-1,0}-\frac{x\left(23+50 x+23 x^{2}\right)}{3(1-x)^{4}} H_{0,1,0,0} \\
& -\frac{4 x\left(25+86 x+25 x^{2}\right)}{9(1-x)^{4}} H_{1,0,-1,0}-\frac{4 x\left(11+x-8 x^{2}-5 x^{3}+8 x^{4}\right)}{3(1-x)^{6}} H_{0,0,0,1} \\
& -\frac{x\left(137+36 x-7 x^{2}-204 x^{3}+17 x^{4}\right)}{9(1-x)^{6}} H_{0,0,0,0} \\
& -\frac{4 x\left(5-12 x-37 x^{2}+36 x^{3}+29 x^{4}\right)}{9(1-x)^{6}} H_{0,0,-1,0}+\frac{2 x\left(19+26 x+19 x^{2}\right)}{9(1-x)^{4}} H_{1,1,0,0} \\
& +\frac{x\left(35+107 x-215 x^{2}-71 x^{3}\right)}{9(1-x)^{5}} H_{1,0,0,0}+\frac{x}{324(1-x)^{2}}\left[3002+5442 H_{1}+18 \pi^{2} H_{1}\right. \\
& \left.+6336 H_{1,1}+2532 L_{\mu}+9 \pi^{2} L_{\mu}+1584 H_{0} L_{\mu}+3168 H_{1} L_{\mu}+792 L_{\mu}^{2}\right] \\
& +\frac{8 x\left(1-4 x+x^{2}\right)}{9(1-x)^{4}}\left[-2 H_{1,0,1,0}-H_{1,0,0} L_{\mu}\right]+\frac{x(1+x)\left(11-8 x+11 x^{2}\right)}{18(1-x)^{5}} \\
& \times\left[-\pi^{2} H_{-1}+12 H_{-1,-1,0}+12 H_{-1,0,1}\right]+\frac{2 x\left(11+13 x+5 x^{2}-5 x^{3}\right)}{3(1-x)^{5}}\left[-2 H_{0,0,1,0}\right. \\
& \left.-H_{0,0,0} L_{\mu}\right]+\frac{x\left(3-4 x+16 x^{2}-4 x^{3}+3 x^{4}\right)}{9(1-x)^{6}}\left[-\pi^{2} H_{0,-1}+12 H_{0,-1,-1,0}\right. \\
& \left.+12 H_{0,-1,0,1}\right]+\frac{x(1+x)^{2}}{1080(1-x)^{4}}\left[-86 \pi^{4} H_{0}-220 \pi^{4} H_{1}-240 \pi^{2} H_{0,0,-1}-64 \pi^{4} L_{\mu}\right. \\
& -585 \pi^{2} H_{0,0,0}-510 \pi^{2} H_{0,0,1}-1230 \pi^{2} H_{0,1,0}-480 \pi^{2} H_{1,0,-1}-1470 \pi^{2} H_{1,0,0} \\
& -960 \pi^{2} H_{1,0,1}-2400 \pi^{2} H_{1,1,0}-10560 H_{0,0,1,1}-10560 H_{0,1,0,1}-10560 H_{0,1,1,0} \\
& -1920 H_{1,0,0,1}+8640 H_{0,0,-1,-1,0}-7200 H_{0,0,-1,0,0}-5760 H_{0,0,-1,1,0}-120 \pi^{2} \zeta_{3} \\
& -8640 H_{0,0,0,-1,0}-2880 H_{0,0,0,0,0}-2160 H_{0,0,0,0,1}-1080 H_{0,0,0,1,0}+3240 H_{0,0,1,0,0} \\
& -5760 H_{0,0,1,-1,0}-14400 H_{0,1,0,-1,0}+5040 H_{0,1,0,0,0}+17280 H_{1,0,-1,-1,0}+720 \zeta_{5} \\
& -14400 H_{1,0,-1,0,0}-11520 H_{1,0,-1,1,0}-28800 H_{1,0,0,-1,0}+7920 H_{1,0,0,0,0} \\
& +5760 H_{1,0,0,1,0}-11520 H_{1,0,1,-1,0}+5760 H_{1,0,1,0,0}-28800 H_{1,1,0,-1,0} \\
& +14400 H_{1,1,0,0,0}-2780 H_{0,0} L_{\mu}-255 \pi^{2} H_{0,0} L_{\mu}-480 \pi^{2} H_{1,0} L_{\mu}-5280 H_{0,0,1} L_{\mu} \\
& -5280 H_{0,1,0} L_{\mu}-2880 H_{0,0,-1,0} L_{\mu}-720 H_{0,0,0,0} L_{\mu}-5760 H_{1,0,-1,0} L_{\mu} \\
& +2880 H_{1,0,0,0} L_{\mu}-1320 H_{0,0} L_{\mu}^{2}-24480 H_{1} \zeta_{3}-9750 H_{0,0} \zeta_{3}-21600 H_{0,1} \zeta_{3} \\
& \left.-21600 H_{1,0} \zeta_{3}-43200 H_{1,1} \zeta_{3}-4320 H_{0} L_{\mu} \zeta_{3}-8640 H_{1} L_{\mu} \zeta_{3}\right] \text {. }
\end{aligned}
$$

The results with an $\overline{\mathrm{MS}}$ renormalized quark mass is

$$
\begin{aligned}
& \overline{\mathcal{C}}_{g g H}^{\left(C_{F}\right)}=\mathcal{C}_{g g H}^{\left(C_{F}\right)}+\Delta \mathcal{C}_{g g H}^{\left(C_{F}\right)}, \\
& \overline{\mathcal{C}}_{g g H}^{\left(C_{A}\right)}=\mathcal{C}_{g g H}^{\left(C_{A}\right)},
\end{aligned}
$$


where

$$
\begin{aligned}
\Delta \mathcal{C}_{g g H}^{\left(C_{F}\right)}=- & \frac{x(3+11 x)}{2(1-x)^{3}} H_{0} L_{\mu}-\frac{x(7+135 x)}{24(1-x)^{3}} H_{0}-\frac{x\left(11+42 x+3 x^{2}\right)}{2(1-x)^{4}} H_{0,0} L_{\mu} \\
- & \frac{x\left(135+426 x+7 x^{2}\right)}{24(1-x)^{4}} H_{0,0}+\frac{x}{12(1-x)^{2}}\left[71+8 \pi^{2}+64 H_{1}+84 L_{\mu}+48 H_{1} L_{\mu}\right. \\
& \left.+36 L_{\mu}^{2}\right]+\frac{x(1+x)}{6(1-x)^{3}}\left[-2 \pi^{2} H_{0}-16 H_{0,1}-16 H_{1,0}-12 H_{0,1} L_{\mu}-12 H_{1,0} L_{\mu}\right. \\
& \left.-9 H_{0} L_{\mu}^{2}\right]+\frac{x\left(1+6 x+x^{2}\right)}{6(1-x)^{4}}\left[-2 \pi^{2} H_{0,0}-24 H_{0,0,0}-16 H_{0,0,1}-16 H_{0,1,0}\right. \\
& \left.-16 H_{1,0,0}-18 H_{0,0,0} L_{\mu}-12 H_{0,0,1} L_{\mu}-12 H_{0,1,0} L_{\mu}-12 H_{1,0,0} L_{\mu}-9 H_{0,0} L_{\mu}^{2}\right] .
\end{aligned}
$$

The contribution $\mathcal{C}_{g g H}^{\left(C_{A}\right)}$ is renormalization scheme independent.

For the sake of completeness, we also provide the $n_{l}^{2}$-contribution $\tilde{C}_{g g H}^{(2,2)}$ originating from the infrared subtraction, cf. eq. (3.9):

$$
\begin{aligned}
& \tilde{C}_{g g H}^{(2,2)}=T_{F}^{3}\left\{\frac { x } { 5 4 ( 1 - x ) ^ { 2 } } \left[-\pi^{2}-40 H_{0}-80 H_{1}-48 H_{0,0}-96 H_{0,1}-96 H_{1,0}-192 H_{1,1}\right.\right. \\
&\left.-40 L_{\mu}-48 H_{0} L_{\mu}-96 H_{1} L_{\mu}-24 L_{\mu}^{2}\right]+\frac{x(1+x)^{2}}{108(1-x)^{4}}\left[\pi^{2} H_{0,0}+120 H_{0,0,0}\right. \\
&+80 H_{0,0,1}+80 H_{0,1,0}+80 H_{1,0,0}+288 H_{0,0,0,0}+288 H_{0,0,0,1}+288 H_{0,0,1,0} \\
&+192 H_{0,0,1,1}+288 H_{0,1,0,0}+192 H_{0,1,0,1}+192 H_{0,1,1,0}+288 H_{1,0,0,0} \\
&+192 H_{1,0,0,1}+192 H_{1,0,1,0}+192 H_{1,1,0,0}+40 H_{0,0} L_{\mu}+144 H_{0,0,0} L_{\mu} \\
&\left.\left.+96 H_{0,0,1} L_{\mu}+96 H_{0,1,0} L_{\mu}+96 H_{1,0,0} L_{\mu}+24 H_{0,0} L_{\mu}^{2}\right]\right\}
\end{aligned}
$$

\section{Ancillary file}

The ancillary file ggh-aah-nl.m contains the main results of this paper in an electronic form readable by Mathematica. ${ }^{4}$ The following table describes its notation:

\begin{tabular}{|ll|}
\hline CaahOS (CaahMSbar) & $C_{\gamma \gamma H}$ \\
Caah0S0 (CaahMSbar0) & $C_{\gamma \gamma H}^{(0)}$ \\
Caah0S1 (CaahMSbar1) & $C_{\gamma \gamma H}^{(1)}$ \\
Caah0S2 (CaahMSbar2) & $C_{\gamma \gamma H}^{(2)}$ \\
\hline
\end{tabular}

\footnotetext{
${ }^{4}$ Wolfram Research, Inc., Mathematica, Version 12.0, Champaign, IL, U.S.A.
} 


\begin{tabular}{|ll|}
\hline caahOS2nl1nonsing (caahMSbar2nl1nonsing) & $\mathcal{C}_{\gamma \gamma H}^{(\text {non-sing) }}$ \\
caah0S2n11sing (caahMSbar2nl1sing) & $\mathcal{C}_{\gamma \gamma H}^{(\text {sing })}$ \\
Cggh0S (CgghMSbar) & $\tilde{C}_{g g H}$ \\
Cggh0S0 (CgghMSbar0) & $\tilde{C}_{g g H}^{(0)}$ \\
Cggh0S1 (CgghMSbar1) & $\tilde{C}_{g g H}^{(1)}$ \\
Cggh0S2 (CgghMSbar2) & $\tilde{C}_{g g H}^{(2)}$ \\
cgghOS2nl1cF (cgghMSbar2nl1cF) & $\mathcal{C}_{g g H}^{\left(C_{F}\right)}$ \\
cggh0S2nl1cA (cgghMSbar2nl1cA) & $\mathcal{C}_{g g H}^{\left(C_{A}\right)}$ \\
\hline api & $\alpha / \pi$ \\
aspi & $\alpha_{s} / \pi$ \\
Lmu & $L_{\mu}$ \\
QQ & $Q_{\mathrm{q}}^{2}$ \\
QQ2 & $\sum_{i=1}^{n_{l}} Q_{i}^{2}$ \\
HPL [a_List, x] & $H_{\vec{a}}$ \\
\hline
\end{tabular}

The OS (MSbar) versions correspond to a quark mass renormalized in the on-shell ( $\overline{\mathrm{MS}})$ scheme. The harmonic polylogarithms are in a format compatible with the Mathematica package HPL.m [56].

Open Access. This article is distributed under the terms of the Creative Commons Attribution License (CC-BY 4.0), which permits any use, distribution and reproduction in any medium, provided the original author(s) and source are credited.

\section{References}

[1] HL/HE WG2 Group, Higgs Physics at the HL-LHC and HE-LHC, arXiv:1902.00134 [INSPIRE].

[2] M. Spira, QCD effects in Higgs physics, Fortsch. Phys. 46 (1998) 203 [hep-ph/9705337] [INSPIRE].

[3] M. Spira, A. Djouadi, D. Graudenz and P.M. Zerwas, Higgs boson production at the LHC, Nucl. Phys. B 453 (1995) 17 [hep-ph/9504378] [INSPIRE].

[4] R.V. Harlander, H. Mantler, S. Marzani and K.J. Ozeren, Higgs production in gluon fusion at next-to-next-to-leading order QCD for finite top mass, Eur. Phys. J. C 66 (2010) 359 [arXiv: 0912.2104] [INSPIRE].

[5] R.V. Harlander and K.J. Ozeren, Finite top mass effects for hadronic Higgs production at next-to-next-to-leading order, JHEP 11 (2009) 088 [arXiv:0909.3420] [INSPIRE]. 
[6] A. Pak, M. Rogal and M. Steinhauser, Finite top quark mass effects in NNLO Higgs boson production at LHC, JHEP 02 (2010) 025 [arXiv:0911.4662] [INSPIRE].

[7] S. Marzani, R.D. Ball, V. Del Duca, S. Forte and A. Vicini, Higgs production via gluon-gluon fusion with finite top mass beyond next-to-leading order, Nucl. Phys. B 800 (2008) 127 [arXiv:0801.2544] [INSPIRE].

[8] LHC Higgs Cross Section Working Group, Handbook of LHC Higgs Cross Sections: 4. Deciphering the Nature of the Higgs Sector, arXiv:1610.07922 [INSPIRE].

[9] C. Anastasiou et al., High precision determination of the gluon fusion Higgs boson cross-section at the LHC, JHEP 05 (2016) 058 [arXiv: 1602.00695] [INSPIRE].

[10] K. Melnikov and A. Penin, On the light quark mass effects in Higgs boson production in gluon fusion, JHEP 05 (2016) 172 [arXiv: 1602.09020] [INSPIRE].

[11] T. Neumann and C. Williams, The Higgs boson at high $p_{T}$, Phys. Rev. D 95 (2017) 014004 [arXiv: 1609.00367] [INSPIRE].

[12] J.M. Lindert, K. Kudashkin, K. Melnikov and C. Wever, Higgs bosons with large transverse momentum at the LHC, Phys. Lett. B 782 (2018) 210 [arXiv:1801.08226] [InSPIRE].

[13] S.P. Jones, M. Kerner and G. Luisoni, Next-to-Leading-Order QCD Corrections to Higgs Boson Plus Jet Production with Full Top-Quark Mass Dependence, Phys. Rev. Lett. 120 (2018) 162001 [arXiv: 1802.00349] [INSPIRE].

[14] T. Neumann, NLO Higgs + jet production at large transverse momenta including top quark mass effects, J. Phys. Comm. 2 (2018) 095017 [arXiv: 1802.02981] [INSPIRE].

[15] R.V. Harlander, T. Neumann, K.J. Ozeren and M. Wiesemann, Top-mass effects in differential Higgs production through gluon fusion at order $\alpha_{s}^{4}$, JHEP 08 (2012) 139 [arXiv: 1206.0157] [INSPIRE].

[16] T. Neumann and M. Wiesemann, Finite top-mass effects in gluon-induced Higgs production with a jet-veto at NNLO, JHEP 11 (2014) 150 [arXiv:1408.6836] [INSPIRE].

[17] F. Caola, J.M. Lindert, K. Melnikov, P.F. Monni, L. Tancredi and C. Wever, Bottom-quark effects in Higgs production at intermediate transverse momentum, JHEP 09 (2018) 035 [arXiv: 1804.07632] [INSPIRE].

[18] J.M. Lindert, K. Melnikov, L. Tancredi and C. Wever, Top-bottom interference effects in Higgs plus jet production at the LHC, Phys. Rev. Lett. 118 (2017) 252002 [arXiv: 1703.03886] [INSPIRE].

[19] R. Harlander and P. Kant, Higgs production and decay: Analytic results at next-to-leading order QCD, JHEP 12 (2005) 015 [hep-ph/0509189] [INSPIRE].

[20] C. Anastasiou, S. Beerli, S. Bucherer, A. Daleo and Z. Kunszt, Two-loop amplitudes and master integrals for the production of a Higgs boson via a massive quark and a scalar-quark loop, JHEP 01 (2007) 082 [hep-ph/0611236] [INSPIRE].

[21] U. Aglietti, R. Bonciani, G. Degrassi and A. Vicini, Analytic Results for Virtual QCD Corrections to Higgs Production and Decay, JHEP 01 (2007) 021 [hep-ph/0611266] [INSPIRE].

[22] J. Fleischer, O.V. Tarasov and V.O. Tarasov, Analytical result for the two loop $Q C D$ correction to the decay $H \rightarrow 2 \gamma$, Phys. Lett. B 584 (2004) 294 [hep-ph/0401090] [INSPIRE]. 
[23] M. Steinhauser, Corrections of $O\left(\alpha_{s}^{2}\right)$ to the decay of an intermediate mass Higgs boson into two photons, in proceedings of the Ringberg Workshop: The Higgs Puzzle - What can We Learn from LEP2, LHC, NLC, and FMC?, Tegernsee, Germany, 8-13 December 1996, pp. 177-185 [hep-ph/9612395] [INSPIRE].

[24] P. Maierhöfer and P. Marquard, Complete three-loop QCD corrections to the decay $H \rightarrow \gamma \gamma$, Phys. Lett. B 721 (2013) 131 [arXiv:1212.6233] [InSPIRE].

[25] R.V. Harlander and K.J. Ozeren, Top mass effects in Higgs production at next-to-next-to-leading order QCD: Virtual corrections, Phys. Lett. B 679 (2009) 467 [arXiv: 0907.2997] [INSPIRE].

[26] A. Pak, M. Rogal and M. Steinhauser, Virtual three-loop corrections to Higgs boson production in gluon fusion for finite top quark mass, Phys. Lett. B 679 (2009) 473 [arXiv: 0907.2998] [INSPIRE].

[27] R. Gröber, A. Maier and T. Rauh, Reconstruction of top-quark mass effects in Higgs pair production and other gluon-fusion processes, JHEP 03 (2018) 020 [arXiv: 1709.07799] [INSPIRE].

[28] J. Davies, R. Gröber, A. Maier, T. Rauh and M. Steinhauser, Top quark mass dependence of the Higgs boson-gluon form factor at three loops, Phys. Rev. D 100 (2019) 034017 [arXiv: 1906.00982] [INSPIRE].

[29] P. Nogueira, Automatic Feynman graph generation, J. Comput. Phys. 105 (1993) 279 [INSPIRE].

[30] R. Harlander, T. Seidensticker and M. Steinhauser, Complete corrections of $O\left(\alpha \alpha_{s}\right)$ to the decay of the $Z$ boson into bottom quarks, Phys. Lett. B 426 (1998) 125 [hep-ph/9712228] [INSPIRE].

[31] T. Seidensticker, Automatic application of successive asymptotic expansions of Feynman diagrams, in proceedings of the 6th International Workshop on New Computing Techniques in Physics Research: Software Engineering, Artificial Intelligence Neural Nets, Genetic Algorithms, Symbolic Algebra, Automatic Calculation (AIHENP 99), Heraklion, Crete, Greece, 12-16 April 1999, hep-ph/9905298 [INSPIRE].

[32] J.A.M. Vermaseren, New features of FORM, math-ph/0010025 [INSPIRE].

[33] T. van Ritbergen, A.N. Schellekens and J.A.M. Vermaseren, Group theory factors for Feynman diagrams, Int. J. Mod. Phys. A 14 (1999) 41 [hep-ph/9802376] [INSPIRE].

[34] F.V. Tkachov, A Theorem on Analytical Calculability of Four Loop Renormalization Group Functions, Phys. Lett. B 100 (1981) 65 [inSPIRE].

[35] K.G. Chetyrkin and F.V. Tkachov, Integration by Parts: The Algorithm to Calculate $\beta$-functions in 4 Loops, Nucl. Phys. B 192 (1981) 159 [InSPIRE].

[36] S. Laporta, High precision calculation of multiloop Feynman integrals by difference equations, Int. J. Mod. Phys. A 15 (2000) 5087 [hep-ph/0102033] [inSPIRE].

[37] P. Maierhöfer, J. Usovitsch and P. Uwer, Kira - A Feynman integral reduction program, Comput. Phys. Commun. 230 (2018) 99 [arXiv:1705.05610] [INSPIRE].

[38] P. Maierhöfer and J. Usovitsch, Kira 1.2 Release Notes, arXiv:1812.01491 [InSPIRE].

[39] A.V. Kotikov, Differential equations method: New technique for massive Feynman diagrams calculation, Phys. Lett. B 254 (1991) 158 [INSPIRE]. 
[40] A.V. Kotikov, Differential equations method: The Calculation of vertex type Feynman diagrams, Phys. Lett. B 259 (1991) 314 [INSPIRE].

[41] A.V. Kotikov, Differential equation method: The Calculation of $N$ point Feynman diagrams, Phys. Lett. B 267 (1991) 123 [Erratum ibid. B 295 (1992) 409] [INSPIRE].

[42] T. Gehrmann and E. Remiddi, Differential equations for two loop four point functions, Nucl. Phys. B 580 (2000) 485 [hep-ph/9912329] [INSPIRE].

[43] J.M. Henn, Multiloop integrals in dimensional regularization made simple, Phys. Rev. Lett. 110 (2013) 251601 [arXiv: 1304.1806] [INSPIRE].

[44] R.N. Lee, Reducing differential equations for multiloop master integrals, JHEP 04 (2015) 108 [arXiv:1411.0911] [INSPIRE].

[45] M. Prausa, epsilon: A tool to find a canonical basis of master integrals, Comput. Phys. Commun. 219 (2017) 361 [arXiv:1701.00725] [INSPIRE].

[46] S.G. Gorishnii, Construction of Operator Expansions and Effective Theories in the MS Scheme, Nucl. Phys. B 319 (1989) 633 [InSPIRE].

[47] V.A. Smirnov, Asymptotic expansions in limits of large momenta and masses, Commun. Math. Phys. 134 (1990) 109 [InSPIRE].

[48] V.A. Smirnov, Asymptotic expansions in momenta and masses and calculation of Feynman diagrams, Mod. Phys. Lett. A 10 (1995) 1485 [hep-th/9412063] [INSPIRE].

[49] V.A. Smirnov, Applied Asymptotic Expansions in Momenta and Masses, Springer Tracts Mod. Phys. 177 (2002) 1 [INSPIRE].

[50] A.V. Smirnov, FIESTA4: Optimized Feynman integral calculations with GPU support, Comput. Phys. Commun. 204 (2016) 189 [arXiv:1511.03614] [INSPIRE].

[51] S. Catani, The Singular behavior of QCD amplitudes at two loop order, Phys. Lett. B 427 (1998) 161 [hep-ph/9802439] [INSPIRE].

[52] D. de Florian and J. Mazzitelli, A next-to-next-to-leading order calculation of soft-virtual cross sections, JHEP 12 (2012) 088 [arXiv: 1209.0673] [INSPIRE].

[53] D. Binosi, J. Collins, C. Kaufhold and L. Theussl, JaxoDraw: A Graphical user interface for drawing Feynman diagrams. Version 2.0 release notes, Comput. Phys. Commun. 180 (2009) 1709 [arXiv: 0811.4113] [INSPIRE].

[54] J.A.M. Vermaseren, Axodraw, Comput. Phys. Commun. 83 (1994) 45 [inSPIRE].

[55] E. Remiddi and J.A.M. Vermaseren, Harmonic polylogarithms, Int. J. Mod. Phys. A 15 (2000) 725 [hep-ph/9905237] [INSPIRE].

[56] D. Maitre, HPL, a mathematica implementation of the harmonic polylogarithms, Comput. Phys. Commun. 174 (2006) 222 [hep-ph/0507152] [INSPIRE]. 